\title{
Correction of lower eyelid retraction in thyroid eye disease: a randomised controlled trial of retractor tenotomy with adjuvant antimetabolite versus scleral graft
}

\author{
Jane M Olver, Geoffrey E Rose, P T Khaw, J R O Collin
}

\begin{abstract}
Backgroundlaims-Lower eyelid retraction in thyroid eye disease contributes to ocular discomfort and an unsightly appearance, especially if asymmetrical. The use of donor scleral grafts is effective in lengthening the lower eyelids but carries a risk of virus transmission. Other techniques, including those which do not use grafts, need to be compared with scleral grafts. Recurrent retraction is a recognised complication of thyroid eyelid surgery; therefore, the authors investigated the use of antimetabolites to reduce postoperative fibrosis.
\end{abstract}

Methods-In this prospective randomised controlled trial of 25 patients ( 35 eyelids), the use of donor sclera in 20 lower eyelids (13 patients) was compared with partial tenotomy of the anterior part of the lower eyelid retractors (ALER) with adjuvant peroperative antimetabolite in 15 lower eyelids (12 patients). A 5 minute peroperative application of either 5-fluorouracil $(25 \mathrm{mg} / \mathrm{ml}$ ) in nine lower eyelids (eight patients) or mitomycin $C(0.2 \mathrm{mg} / \mathrm{ml})$ in six lower eyelids (four patients) was used to focally inhibit fibroblasts. Follow up ranged from 3 to 18 months (mean 7.8). Results-One month after surgery the results of both groups were similar. However, at 3 months after surgery the results of scleral grafting were better than tenotomy with antimetabolites: $3 / 12$ patients $(25 \%)$ treated with tenotomy and adjuvant antimetabolite required subsequent surgery using grafts for correction of recurrent retraction. There were no significant complications associated with the use of antimetabolites in the eyelid in the doses used in this study.

Conclusions-This randomised prospective trial shows that donor scleral grafts were more effective in the long term than partial tenotomy with adjuvant antimetabolite in the correction of lower eyelid retraction associated with thyroid eye disease. The use of peroperative antimetabolites in the lower eyelid was safe. (Br F Ophthalmol 1998;82:174-180)

Lower eyelid retraction may occur in thyroid eye disease secondary to proptosis, lower eyelid retractor shortening (fibrosis), and previous inferior rectus muscle recession. Surgery to correct lower eyelid retraction may include either a graft or a non-graft technique. ${ }^{1-24}$ Grafts may be autogenous-for example, as hard palate mucosa, ${ }^{13} 1520$ auricular cartilage, ${ }^{14}$ tarsal conjunctiva, ${ }^{21}{ }^{24}$ and buccal mucosa, ${ }^{25}$ or may be from an exogenous donor such as sclera. Donor sclera has a risk of transmission of HIV or slow viruses, may be of limited availability and may not be acceptable to the patient for ethical reasons. Synthetic graft materials such as polytetrafluoroethylene (Gortex) and polyester mesh (Mersilene) have been used but have not gained general acceptance. Non-graft techniques $^{14817}$ to raise the lower eyelid consist of recession, tenotomy, or extirpation of the anterior part of the lower eyelid retractors (ALER) - that is, the capsulopalpebral fascia and inferior tarsal muscle. ${ }^{26}{ }^{27}$ There have been no prospective randomised trials comparing these different surgical techniques.

After surgery for lower eyelid retraction, fibrosis may cause a recurrence of the retraction and, therefore, inhibition of fibrosis might prevent recurrent retraction. A single 5 minute exposure of a cell culture to 5-fluorouracil or mitomycin $\mathrm{C}$ inhibited human Tenon's capsule fibroblast proliferation for at least 36 days. $^{28}$ Likewise, a single 5 minute intraoperative application of 5-fluorouracil $(50 \mathrm{mg} / \mathrm{ml})$ resulted in reversible delay of fibroblast inhibition in rabbit Tenon's capsule, and mitomycin C $(0.4 \mathrm{mg} / \mathrm{ml})$ resulted in prolonged inhibition, for 30 days. $^{29}$

In this era of evidence based medicine we are required to demonstrate in a scientific way that new techniques are better than the best current practice (even in oculoplastics). ${ }^{30}$ We have therefore performed a randomised controlled prospective trial; lower eyelid lengthening with a well established technique, scleral graft interposition without adjuvant antimetabolites, was compared with partial tenotomy of the capsulopalpebral fascia augmented with a single peroperative application of adjuvant 5-fluorouracil or mitomycin $\mathrm{C}$ to reduce postoperative fibrosis.

\section{Methods}

Consecutive patients with lower eyelid retraction requiring surgical correction were recruited into the study from the thyroid and adnexal clinics at Moorfields Eye Hospital. Ethics committee permission was obtained and each patient gave informed consent to enter the study and be randomised prospectively to one of the two treatment groups. If the lower 
eyelid retraction was bilateral, each eyelid received the same surgical treatment. Every patient was euthyroid and had stable thyroid eye disease for at least 6 months before eyelid surgery. The indications for surgery were for improvement of cosmetic appearance (particularly for asymmetry), ocular discomfort, and lagophthalmos.

Twenty female and five male patients (35 eyelids) were recruited into the study, with an age range of 37-73 years (mean 57, median 56). Four patients (seven orbits) had earlier two wall decompressions and 13 patients (19 eyes) had previous strabismus surgery, including inferior rectus recession of $4-6 \mathrm{~mm}$, without simultaneous lower eyelid retractor surgery.

Clinical measurements of palpebral aperture, lower margin reflex distance (MRD), lower scleral show (SS), and photographic documentation of each patient were made before surgery and at fixed intervals ( 1 week and at $1,3,6,9,12$, and 18 months) after surgery. The lower MRD being the distance (in $\mathrm{mm}$ ) from a single central corneal light reflex to the lower eyelid margin, and the lower SS the distance from the inferior limbus to the lower eyelid margin, both measured with the patient sitting with head straight and eye position in primary gaze. The lower eyelid contour was also recorded.

Before surgery by a single surgeon (JMO), the patients were randomised to receive donor scleral graft (group A) or to have a partial tenotomy of the anterior part of the lower eyelid retractors-ALER-(capsule-palpebral fascia and inferior tarsal muscle) with peroperative adjuvant antimetabolite (group B).

GROUP A SURGERY: DONOR SCLERA

Donor sclera was obtained from donors without known neurological disease and tested negative for HIV and hepatitis B, prepared in alcohol and washed before use. Surgery was performed under either local anaesthetic infiltration (bupivacaine $0.5 \%$ with adrenaline $1: 100000)$ or general anaesthesia, according to the patient's preference. Two 4/0 nylon traction sutures were placed in the lower eyelid margin and the eyelid everted over a Desmarres' retractor. The conjunctiva and the anterior part of the lower eyelid retractors (ALER) were divided from the lower border of the tarsal plate, dissected off the orbital septum and orbicularis anteriorly, and the ALER separated from the conjunctiva. Alternatively, the conjunctiva was initially dissected free of the underlying ALER and then the lower eyelid retractors disinserted from the tarsal edge. The medial and lateral horns of the ALER were divided. The prepared scleral graft (approximately 7-9 $\mathrm{mm}$ height and $22-23 \mathrm{~mm}$ length) was interposed between the retractors and lower border of the tarsal plate, covered by the conjunctiva. The vertical height of the scleral graft size was selected as $6 \mathrm{~mm}$ greater than the lower scleral show; this extra $6 \mathrm{~mm}$ allowing 3-4 mm loss of height due to suturing and 2-3 $\mathrm{mm}$ loss due to postoperative fibrosis.

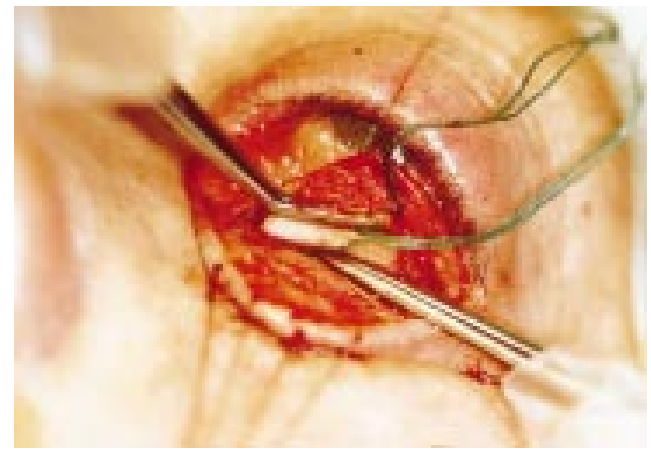

Figure 1 The adjuvant antimetabolite is applied focally to each side of the anterior part of the lower eyelid retractors (ALER) consisting of the capsulopalpebral fascia and inferior tarsal muscle. Two soaked neurosurgical patties (with radio-opaque tie) are used. The cut upper end of the ALER is held in a muscle clamp and is not treated with antimetabolite.

The lower border of the graft was sutured to the upper edge of the retractors with continuous $6 / 0$ Vicryl (Ethicon Ltd) and, to close the incision, the upper border of the scleral graft sutured to the edge of the conjunctiva and lower border of tarsal plate with continuous $7 / 0$ Vicryl. A 2-3 mm lateral tarsorrhaphy was fashioned where indicated (for example, with asymmetrical proptosis and lower eyelid retraction and with bilateral proptosis $>25 \mathrm{~mm}$ ). The lower eyelid was placed on upward traction and firmly padded overnight. No bolsters were placed through the graft, except where lower scleral show exceeded $3 \mathrm{~mm}$ preoperatively and the graft was of greater than $9 \mathrm{~mm}$ height.

\section{GROUP B SURGERY: PARTIAL TENOTOMY AND} ADJUVANT ANTIMETABOLITE

Under local or general anaesthesia, the anterior part of the lower eyelid retractors (ALER) was isolated and the upper edge held in a ptosis clamp. The conjunctiva was reflected back over the cornea and a neurosurgical pattie $(12.5 \times 6$ $\mathrm{mm}$ ), soaked in antimetabolite, was placed each side of the lower eyelid retractors for 5 minutes, care being taken to avoid the edge held in the ptosis clamp, the conjunctival edge, or the ocular surface (Fig 1). After removal of the patties, the treated area was irrigated with $10 \mathrm{ml}$ saline and the instruments changed before completion of the procedure. Partial tenotomies were performed by making extensive lateral, medial, and central incisions into the ALER, to significantly lengthen it. Using a continuous $7 / 0$ Vicryl, the upper edge of the ALER retractors was sutured to the lower border of the tarsal plate and the cut edge of the conjunctiva. As with group A, where necessary, a 2-3 $\mathrm{mm}$ lateral tarsorrhaphy was fashioned. Vertically placed silicone bolsters kept the lower eyelid stretched upwards for approximately 2 weeks after surgery. These were sutured in place with 4/0 nylon through the skin, orbicularis, and lengthened ALER, but not through the conjunctiva.

Either 5-fluorouracil (David Bull Laboratories) or mitomycin C (Kyowa) was used in group $\mathrm{B}$, the first eight patients (nine lower eyelids) receiving $0.4 \mathrm{ml} 5$-fluorouracil $(25 \mathrm{mg}$ / $\mathrm{ml}$ ) and the next four patients (six lower 
Table 1 Preoperative characteristics of 25 patients undergoing surgery for dysthyroid lower eyelid retraction

\begin{tabular}{lll}
\hline & Group $A$-scleral graft & $\begin{array}{l}\text { Group B-tenotomy and } \\
\text { antimetabolite }\end{array}$ \\
\hline No of patients & 13 & 12 \\
Sex & 9 female, 4 male & 11 female, 1 male \\
Age at surgery & $40-73$ years (mean 54) & $37-73$ years (mean 59) \\
Previous inferior rectus recession & 12 eyelids (8 patients) & 7 eyelids (5 patients) \\
Number of eyelids & 20 & 15 \\
Laterality & 7 bilateral, 6 unilateral & 3 bilateral, 9 unilateral \\
Mean Rodenstock exophthalmometry & $22.6 \mathrm{~mm}$ & $22.4 \mathrm{~mm}$ \\
$\quad$ Range & $17-28 \mathrm{~mm}$ & $17-30 \mathrm{~mm}$ \\
Mean lower lid margin reflex distance (SD) & $7.5 \mathrm{~mm}(0.9)$ & $7.9 \mathrm{~mm}(0.8)$ \\
$\quad$ Range & $5-9 \mathrm{~mm}$ & $7-9 \mathrm{~mm}$ \\
Mean inferior (lower) scleral show (SD) & $2.5 \mathrm{~mm}(1.0)$ & $2.9 \mathrm{~mm}(0.8)$ \\
$\quad$ Range & $1-4 \mathrm{~mm}$ & $1-4 \mathrm{~mm}$ \\
\hline
\end{tabular}

Table 2 Preoperative characteristics of patients undergoing tenotomy with adjuvant antimetabolite (group B)

\begin{tabular}{lll}
\hline & \multicolumn{2}{l}{ Type of antimetabolite } \\
\cline { 2 - 3 } & 5-fluorouracil & Mitomycin $C$ \\
\hline No of patients & 8 & 4 \\
Sex & 7 female, 1 male & 4 female \\
Age at surgery & $42-73$ years (mean 59) & $52-70$ years (mean 58) \\
Number of eyelids & 9 bilateral, 7 unilateral & 6 \\
Laterality & 12 bilateral, 2 unilateral \\
Mean Rodenstock exophthalmometry & $22 \mathrm{~mm}$ & $23 \mathrm{~mm}$ \\
Range & $17-28 \mathrm{~mm}$ & $19-25 \mathrm{~mm}$ \\
Mean lower lid margin reflex distance (SD) & $7.9 \mathrm{~mm}(0.79)$ & $8 \mathrm{~mm}(0.89)$ \\
Range & $7-9 \mathrm{~mm}$ & $7-9 \mathrm{~mm}$ \\
Mean inferior (lower) scleral show (SD) & $2.78 \mathrm{~mm}(0.67)$ & $3 \mathrm{~mm}(0.63)$ \\
Range & $2-4 \mathrm{~mm}$ & $2-4 \mathrm{~mm}$ \\
\hline
\end{tabular}

Table 3 Characteristics of lower eyelid positon before and after surgery

\begin{tabular}{lll}
\hline Time after surgery & $\begin{array}{l}\text { Scleral grafting } \\
\text { (group A; 20 eyelids) }\end{array}$ & $\begin{array}{l}\text { Tenotomy and antimetabolite } \\
\text { (group B; 15 eyelids) }\end{array}$ \\
\hline Mean lower margin reflex distance $(\mathrm{mm}):$ & $7.5 \mathrm{~mm}(0.95)$ & $7.9 \mathrm{~mm}(0.8)$ \\
Preoperative (SD) & $5-9 \mathrm{~mm}$ & $7-9 \mathrm{~mm}$ \\
Range & $5.75(\mathrm{~S} . \mathrm{D} .1 .02)^{\star}$ & $6.8(\mathrm{~S} . \mathrm{D} .1 .15)^{\star \star}$ \\
1 month (SD) & $4-7 \mathrm{~mm}$ & $4-8 \mathrm{~mm}$ \\
Range & $6.25(0.88)^{\star}$ & $7.4(1.18)^{\star \star \star}$ \\
3 months (SD) & $4-8 \mathrm{~mm}$ & $6-9 \mathrm{~mm}$ \\
Range & $6.3(0.87)^{\star}$ & $7.29(1.14)^{\star \star \star}$ \\
6 months (SD) & $4-8 \mathrm{~mm}$ & $6-9 \mathrm{~mm}$ \\
Range & $2.45(1.05)$ & $2.87(0.64)$ \\
Mean lower scleral show $(\mathrm{mm}):$ & $1-5 \mathrm{~mm}$ & $2-4 \mathrm{~mm}$ \\
Preoperative (SD) & $0.74(0.70)^{\star}$ & $1.33(1.11)^{\star}$ \\
Range & $0-2 \mathrm{~mm}$ & $0-4 \mathrm{~mm}$ \\
1 month (SD) & $0.87(0.74)^{\star}$ & $1.93(1.39)^{\star \star \star}$ \\
Range & $0-2 \mathrm{~mm}$ & $0-4 \mathrm{~mm}$ \\
3 months (SD) & $0.93(0.59)^{\star}$ & $2.4(1.4)^{\star \star \star}$ \\
Range & $0-2.5 \mathrm{~mm}$ & $0-4 \mathrm{~mm}$ \\
6 months (SD) & & \\
Range & & \\
\hline${ }_{\mathrm{p}}<0.001, \star \star \mathrm{p}<0.01, \star \star \star \mathrm{p}>0.05$ with & respect to preoperative values.
\end{tabular}

${ }^{\star} \mathrm{p}<0.001,{ }^{\star \star} \mathrm{p}<0.01,{ }^{\star \star \star} \mathrm{p}>0.05$ with respect to preoperative values.

eyelids) receiving mitomycin $\mathrm{C}(0.2 \mathrm{mg} / \mathrm{ml})$. Mitomycin $\mathrm{C}$ was prepared in water.

DATA COLLECTION AND STATISTICAL ANALYSIS Follow up ranged from 3 to 18 months (mean 7.8, median 7).

Data were collated using Paradox 3.5 and analysis was by use of the Student's $t$ test.

\section{Results}

PREOPERATIVE DATA

There was no significant difference between the patients in group A (scleral graft) and in group B (partial tenotomy and adjuvant antimetabolite $)$ for age $(p=0.81)$, amount of proptosis $(p=0.90)$, preoperative lower MRD $(\mathrm{p}=0.16)$, and preoperative lower SS $(\mathrm{p}=0.16)$ (Table 1). Nine patients (13 eyes) in group A and four patients (six eyes) in group B had prior inferior rectus recession, without retractor recession.

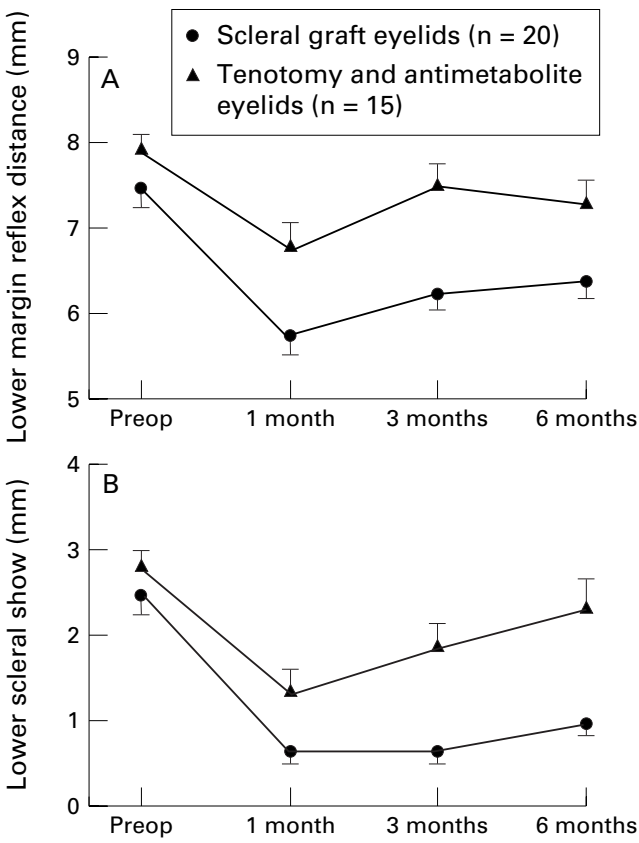

Figure 2 (A) Change in lower margin reflex distance (mean (SEM); $m$ m) after surgery. (B) Change in lower scleral show (mean (SEM); $m m$ ) after surgery.

The patients in group B who had adjuvant 5-fluorouracil and those who had mitomycin C were similar with respect to age $(p=0.81)$, amount of proptosis $(\mathrm{p}=0.57)$, preoperative lower MRD ( $\mathrm{p}=0.80)$, and preoperative lower SS $(\mathrm{p}=0.53)$ (Table 2).

No analysis was made of the vertical palpebral aperture measurements, because several patients had upper eyelid surgery during the study period, thus altering the overall vertical palpebral aperture, but not the lower MRD or the lower SS.

EFFECT OF SURGERY ON THE LOWER EYELID

HEIGHT AND CONTOUR

Group A (scleral graft)

All 13 patients (20 eyelids) had a significant reduction in lower MRD (mean reduction of $1.8 \mathrm{~mm}$; $\mathrm{p}<0.001$; Table 3) and a good eyelid contour 1 month after surgery. The reduction in lower MRD and good contour was maintained at 3 and 6 months after surgery $(p<0.001$; Table 3, Fig 2A), and none required further surgery (Fig 3).

Scleral show was significantly reduced at 1 month $(\mathrm{p}<0.001), 3$ months $(\mathrm{p}<0.001)$, and 6 months after surgery $(p<0.001)$ (Table 3, Fig 2B).

\section{Group B (partial tenotomy and adjuvant} antimetabolite)

The lower MRD was reduced significantly at 1 month after surgery $(p=0.006)$, but was not maintained at 3 months $(p=0.16)$ or 6 months after surgery $(p=0.10)$ (Table 3$)$. Likewise, lower scleral show was reduced significantly at 1 month after surgery $(p<0.001)$, but not maintained at 3 months $(p=0.12)$, or 6 months after surgery $(p=0.22)$ (Table 3 , Fig $2 A$ and $B)$.

There was no significant difference in the change of lower MRD and SS between patients 

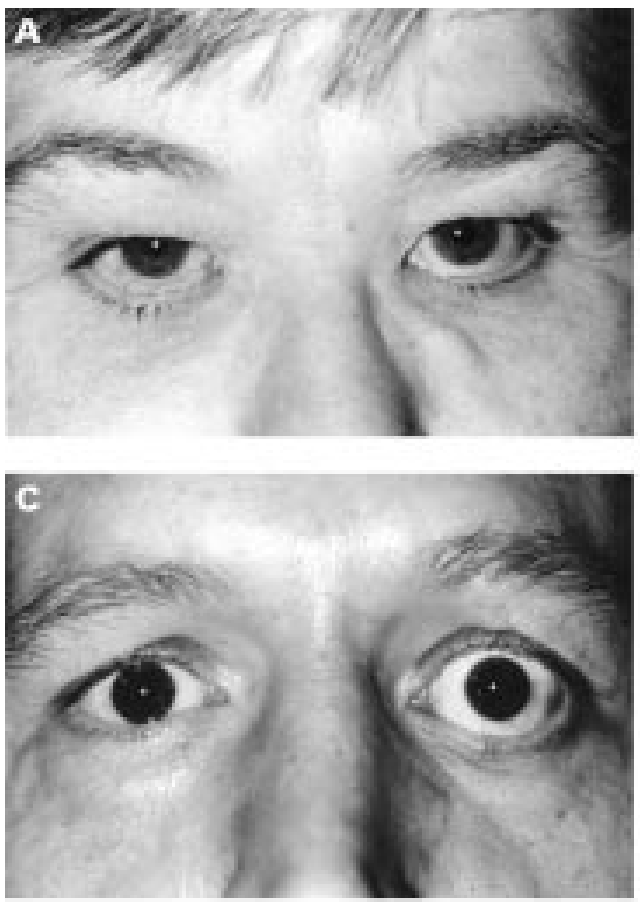

Figure 3 Scleral grafts. (A) Case 1. Bilateral lower eyelid retraction in a patient who has had previous bilateral orbital decompression with later right medial and inferior rectus recession. She also has upper eyelid dermatochalasis which, combined with the lower eyelid retraction, has given her an apparent head tilt with chin down. (B) Case 1. One year after bilateral lower eyelid scleral grafts and upper eyelid blepharoplasty. The upper eyelid blepharoplasty has greatly improved her overall appearance. (C) Case 2. Left unilateral proptosis and marked lower eyelid retraction in a patient who has had previous inferior rectus recession. He had a variable mild left upper eyelid retraction and opted for lower eyelid surgery only. (D) Case 2. After a large sized scleral graft with vertical bolsters. (E) Case 2. Six months after left lower eyelid scleral graft. Note slightly bulky lower eyelid and skin crease lower than the unoperated right side.

treated with adjuvant 5-fluorouracil or mitomycin C.

Four eyelids (three patients) required further lower eyelid lengthening surgery because of recurrent retraction (Fig 4), whereas five eyelids (four patients) had acceptable lower eyelid height, but with a slight central sag to the eyelid contour which might have benefited from further surgery. The results were satisfactory in six eyelids (five patients).

Of the four eyelids requiring further eyelid lengthening surgery, three had received mitomycin $\mathrm{C}$ and one had received 5-fluorouracil.

\section{Postoperative complications}

There were no eyelid or ocular surface complications in patients with donor scleral graft, the eyelids showing only slight inflammation after surgery. The eyelids were more bulky than those having partial tenotomy, and the position of the lower skin crease often increased to more than $4 \mathrm{~mm}$ below the lash line (Fig 3).

One patient who had bilateral partial tenotomy and adjuvant mitomycin $\mathrm{C}$ developed a unilateral corneal epithelial erosion which healed within 48 hours, whereas another patient developed a temporary adhesion between the lower eyelid and the bulbar conjunc-
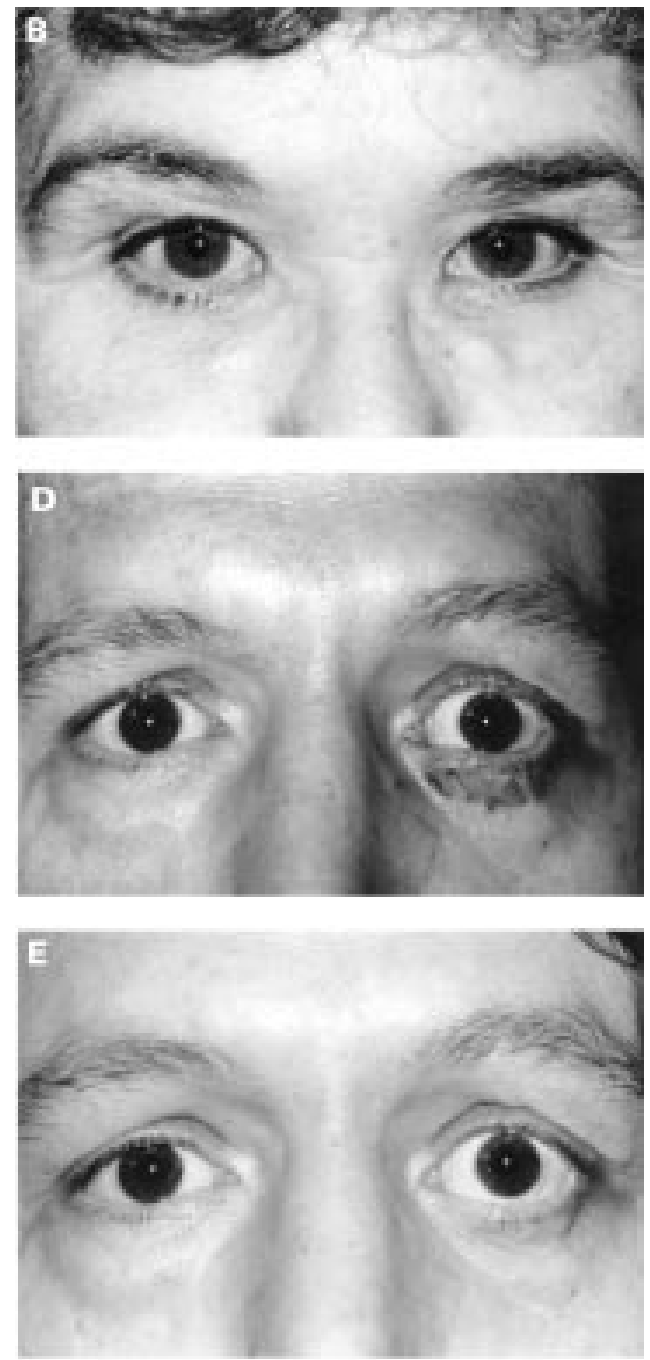

tiva, present at the site of a $1 \mathrm{~mm}$ diameter conjunctival tear, this resolving spontaneously once inflammation had settled. Although postoperative inflammation settled within 4 to 6 weeks, there appeared to be more lower eyelid inflammation after adjuvant antimetabolites, particularly mitomycin $\mathrm{C}$, than with donor scleral graft.

\section{Discussion}

Thyroid eye disease is cosmetically and psychologically debilitating and poses a threat to sight. Lower eyelid retraction may occur due to shortening of the lower eye retractors, both the anterior and posterior parts, accentuated by proptosis and by previous inferior rectus recession. Eyelid retraction increases the vertical palpebral aperture and, hence, the area of exposed ocular surface which may lead to lagophthalmos, ocular discomfort, photophobia, and watering. Although lubricants may partially alleviate symptoms, surgery is often indicated both to relieve symptoms and to improve the patients' cosmetic appearance. Lower eyelid surgery is usually performed after correction of upper eyelid retraction.

The lower eyelid retractors consist of the capsulopalpebral fascia, the inferior tarsal 

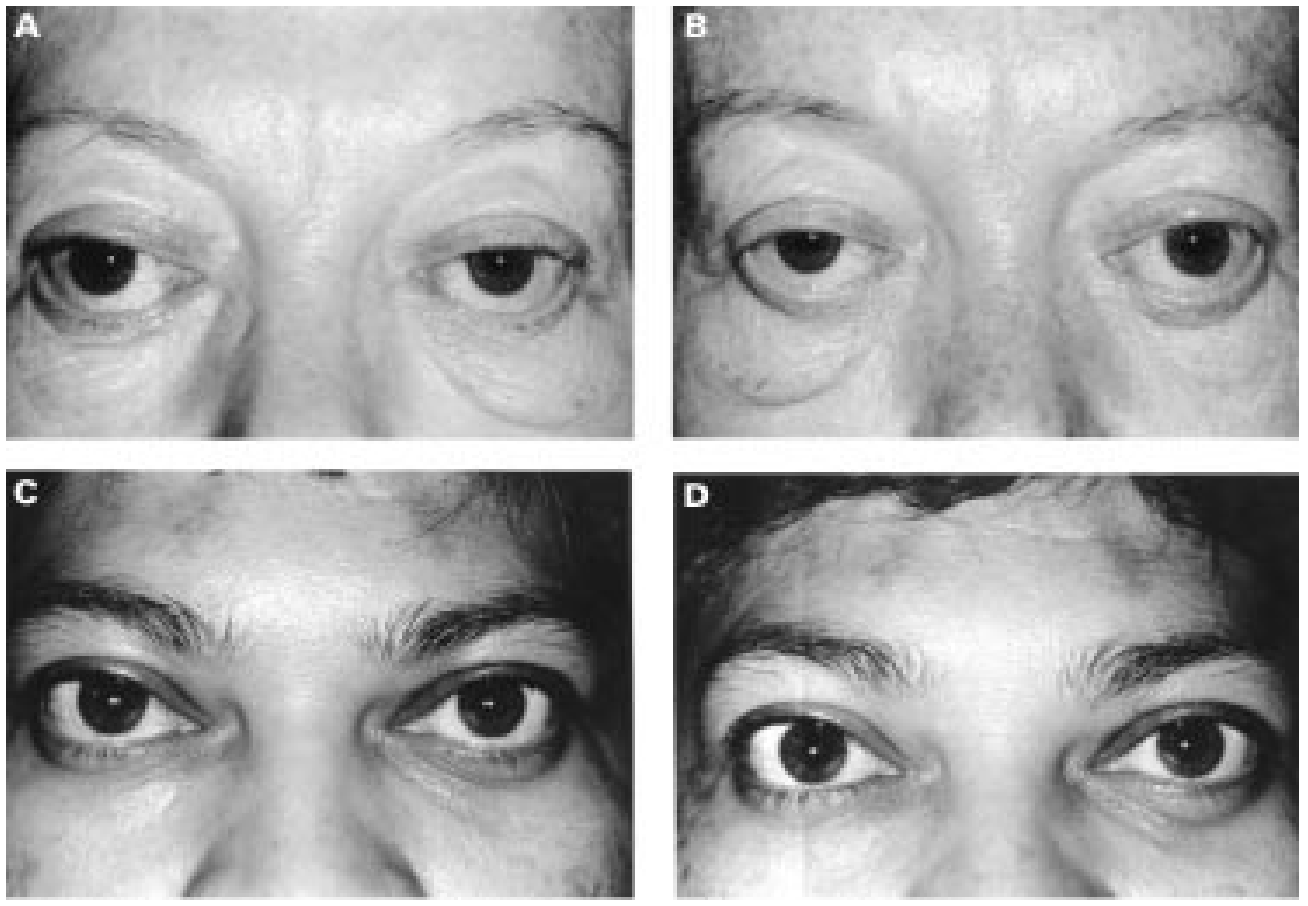

Figure 4 Tenotomy and antimetabolite. (A) Case 3. Bilateral lower eyelid retraction in a thyroid patient who has had previous bilateral upper eyelid retraction surgery (levator recessions) and inferior rectus recession. (B) Case 3. Three months after bilateral lower eyelid partial tenotomy retractors and adjuvant antimetabolite (mitomycin $C$ ), there is no sustained improvement in the lower lid position. This patient subsequently had bilateral palatal mucosal grafts inserted into her lower eyelids. (C) Case 4. Bilateral lower lid retraction before tenotomy and adjuvant 5-fluorouracil. (D)Case 4. Level of lower eyelid maintained 6 months postoperatively.

muscle (equivalent to the Muller's muscle of the upper eyelid), the capsulopalpebral head and Lockwood's ligament with their attachments to the inferior rectus and inferior oblique muscle. ${ }^{26}{ }^{27}$ The anterior part of the lower eyelid retractors (ALER), the capsulopalpebral fascia, and inferior tarsal muscle, are usually lengthened with or without a "spacer" graft. Other techniques have been described in which the capsulopalpebral head is detached from the inferior rectus muscle and advanced on fixed or adjustable sutures ${ }^{21} 22$; this latter approach having been advocated to reduce lid retraction after inferior rectus recession. Non-graft techniques, such as a free tenotomy, sutured recession, or extirpation of the lower eyelid retractors, have not been systematically evaluated or compared with graft techniques and have, to date, been used only to correct small amounts of lower eyelid retraction. ${ }^{14817}$ In this trial we chose to compare a non-graft technique, augmented by peroperative antimetabolite application, with donor scleral grafts.

The rationale for the use of antimetabolites was as follows. Recurrence of retraction after graft surgery is due to graft absorption and fibrosis; that after non-graft techniques may be due to a lack of rigidity in the middle lamella as well as fibrous contracture. After partial tenotomy of the lower eyelid retractors we sought to eliminate or reduce postoperative fibrosis by the use of adjuvant antimetabolites, 5 -fluorouracil $(25 \mathrm{mg} / \mathrm{ml})$ or mitomycin-C $(0.2$ $\mathrm{mg} / \mathrm{ml})$, and determine whether this technique could be as effective as scleral grafting.

Fluorouracil $(25 \mathrm{mg} / \mathrm{ml})$ reversibly inhibits fibroblasts during the first couple of weeks after focal exposure and mitomycin C (0.2 $\mathrm{mg} / \mathrm{ml}$ ) has a more prolonged effect in vitro; the results of this present study were similar in the two groups of antimetabolites although the numbers analysed are small. Mitomycin C at $0.4 \mathrm{mg} / \mathrm{ml}$ was not used since it causes widespread cell death for several months (such higher concentrations might be unsafe in the eyelid $^{31}$ ) and it has been associated with greater complications after glaucoma filtration surgery. ${ }^{32}$

In this trial, the early results ( 1 month) were very similar for the two treatment groups; however, 3 months after surgery, the results of grafting with donor sclera were superior to partial tenotomy with adjuvant antimetabolite. All the patients in this trial had a minimal follow up of 3 months which we believe is important to detect changes in the height of the lower eyelid from fibrosis.

Donor sclera cannot intrinsically contract unless it is coagulated at high temperature, or is invaded by cells; it should, therefore, remain to act as a very useful semirigid spacer. In this trial, antimetabolite was not used in conjunction with scleral graft. In the partial tenotomy group, it was assumed that a 5 minute peroperative focal application of antimetabolite to both sides of the ALER would result in prolonged, but not indefinite, inhibition of fibroblasts, and therefore reduce but not abolish recurrent lower eyelid retraction. It is of note that nine patients (75\%) had acceptable results, which in itself could seem reasonable if it had not been compared in a randomised prospective trial with scleral grafts.

The eyelid contour in those patients who received a donor scleral graft remained almost 
straight horizontally, presumably, due to the stiffness of the graft providing a rigid scaffold. In contrast, there was a dip in the central contour in those patients with recurrent retraction after partial tenotomy and antimetabolite, this was probably due to fibrosis after the effect of antimetabolite had diminished; a positive lower lid traction test or histopathology of the lower lid retractors might provide evidence of the mechanism of recurrent retraction. It is possible that repeated transconjunctival or transcutaneous injections of antimetabolite at 2 and 4 weeks after surgery might have prevented recurrent retraction by further inhibiting fibroblasts, although the central sag may still have occurred because of the lack of a rigid middle lamella and the effect of gravity.

There were no significant complications with either surgical procedure. The eyelids which had received scleral grafts were a bit bulkier and the skin crease lower in comparison with the tenotomy/antimetabolite patients. We documented only minor problems with the use of focal single peroperative antimetabolites, suggesting that judicial use of antimetabolites in oculoplastic surgery is safe.

There are few reports on the use of non-graft techniques to correct lower eyelid retraction in thyroid eye disease. Correction of retraction with simple retractor recession with lateral canthal support $\mathrm{t}^{17}$ is a simple local anaesthetic procedure suitable for small amounts of retraction, but is unpredictable because of fibrosis. A mid-face lift ${ }^{33}$ would augment simple retractor recession and support the lower lid long term in some patients, but is a more extensive operation. Alternatives to retractor recession include retractor extirpation, which would remove a significant source of fibroblasts but is a more destructive procedure which results in conjunctival/orbicularis adhesion.

The use of donor scleral grafts ${ }^{2-101618}$ to lengthen the ALER in eyelid retraction associated with thyroid eye disease is well established for all degrees of lower eyelid retraction and has good results. Surgery may be done under local anaesthesia and the grafts are tolerated well. There may be recurrences of lower eyelid retraction (3-13\%) requiring further surgery, attributed to graft absorption and perigraft fibrosis. Mourits and Koorneef found 27/30 lower eyelid scleral grafts resulted in a good or acceptable result after one operation when assessed 3 months after surgery. Three eyelids $(10 \%)$ required further surgery because of a poor result. Mourits and Koorneef allude to later retraction within 1 year in two patients, a drift which was also noted from our measurement even in the scleral graft group. It has been suggested that better results are obtained long term when a lateral tarsal strip and tarsorrhaphy are used in conjunction with a scleral graft. ${ }^{19}$

Despite the excellent overall results with sclera, the risk of virus transmission has led to a search for alternative suitable grafts. Synthetic graft material such as polytetrafluoroethylene $(\text { Gortex })^{11}$ and polyester mesh

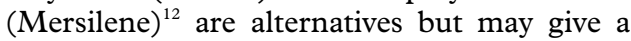
thickened inflamed lower eyelid with a risk of graft extrusion and infection, and therefore have not gained widespread use.

Autogenous hard palate mucosa grafts were first used to reconstruct the posterior lamella of the eyelid after tumour excision then used for the correction of cicatricial entropion with good results. ${ }^{13}$ Hard palate mucosa grafts are composite grafts providing both middle lamella support and posterior lamella mucosa. Their use for the correction of lower eyelid retraction associated with thyroid eye disease has many advantages over donor scleral grafts since the risk of virus transmission is abolished, and shrinkage is minimal $(<10 \%) .{ }^{15} 20$ The grafts may also result in a low skin crease and be bulky in some patients. The main disadvantage is that hard palate mucosa is best harvested with the patient sedated or under general anaesthesia, therefore may not be suitable for all patients. Buccal mucosa grafts ${ }^{25}$ may be more easily harvested under local anaesthesia but do not provide structural support per se in the lower lid. Other autogenous grafts include auricular cartilage, ${ }^{14}$ which may be harvested in small amounts from the concha under local anaesthesia, and free tarsoconjunctival grafts $^{2124}$ which are also easily harvested, but healing of the donor site may theoretically exacerbate upper eyelid retraction.

In this study, consistently satisfactory lower eyelid lengthening, with a good eyelid contour, was obtained only by the use of donor scleral grafts, whereas partial tenotomy and adjuvant peroperative antimetabolite did not give good long term results in $25 \%$ patients. Grafts are still better than antimetabolite augmented tenotomy. Where the use of donor sclera is not desirable or possible, the use of autogenous grafts, such as hard palate mucosa graft or auricular cartilage, are suitable alternatives.

Supported in part by the Medical Research Council Grant Number G9339970. We thank Mr Peter Fells who ran the thyroid clinic at Moorfields Eye Hospital for kindly allowing us to operate on his patients.

Presented at the 13th Meeting of the European Society of Ophthalmic Plastic and Reconstructive Surgery, Rostock, Germany, 31 May-1 June 1995.

1 Henderson JW. Relief of eyelid retraction. A surgical procedure. Arch Ophthalmol 1965;74:205-16.

2 Flanagan JC. Eye bank sclra in oculoplastic surgery. Ophthalmic Surg 1974;5:45-53.

3 Dryden RM, Sol DB. The use of scleral transplantation in cicatricial entropion and eyelid retraction. Ophthalmology 1977;83:669-78.

4 Waller RR. Lower eyelid retraction: management. Ophthalmic Surg 1978;9:41-7.

5 Cooper WC. The surgical management of the lid changes of Graves' disease. Ophthalmology 1979;86:2071-80.

6 Callahan MA, Callahan A. Surgery for endocrine ophthalmopathy. In: Callahan MA, Callahan A, eds. Ophthalmic plastic and orbital surgery. Alabama: Aesculapius, 1979:146.

7 plastic and orbital surgery. Alabama: Aesculapius, 1979:146. Doxanas MT, Dryden RM. The use of sclera in the
treatment of dysthyroid eyelid retraction. Ophthalmology treatment of dysthy

8 Waller RR, Samples JR, Yeatts RP. Eyelid malposition in Graves' ophthalmopathy. In: Gorman AL, et al. The eye and orbit in thyroid disease. 1st ed. NewYork: Raven Press, 1984: 263-300.

9 Shorr N, Seiff SR. The four stages of surgical rehabilitation of the patient with dysthyroid ophthalmopathy. Ophthalmology 1986;93:476-83.

10 Thaller VT, Kaden K, Lane CM, Collin JRO. Thyroid lid surgery. Eye 1987;1:609-14.

11 Karesh JW, Fabrega MA, Rodrigues MM, Glaros DS. Polytetrafluoroethylene as an interpositional graft material for the correction of lower eyelid retraction. Ophthalmology 1989;96:419-23.

12 Downes RN, Jordan K. The surgical management od dysthyroid related eyelid retraction using mersilene mesh. Eye 1989;3:385-90. 
13 Bartley GB, Kay PP. Posterior lamellar eyelid reconstruction with a hard palate mucosal graft. Am $\mathcal{f}$ Ophthalmol with a hard pala

14 Marks MW, Argenta LC, Friedman RJ, Hall JD. Concha cartilage and composite grafts for correction of lower eyelid retraction. Plast Reconstr Surg 1989;83:629-35.

15 Kersten RC, Kulwin DR, Levartovsky S, Tiradellis H, Tse DT. Management of lower-lid retraction with hard-palate mucosa grafting. Arch Ophthalmol 1990;108:1339-43.

16 Hurwitz JJ, Archer KF, Gruss JS. Treatment of severe lower eyelid retraction with scleral and free skin grafts and bipedicle orbicularis flap. Ophthalmic Surg 1990;21:16772 .

17 Holds JB, Anderson RL, Thiese SM. Lower eyelid retraction: a minimal incision surgical approach to retractor lysis. Ophthalmic Surg 1990;21:767-71.

18 Mourits $\mathrm{M} \mathrm{Ph}$, Koorneef L. Lid lengthening by scleral interposition for eyelid retraction in Graves' ophthalmopathy. Br f Ophthalmol 1991;75:344-7.

19 Feldman KA, Putterman AM, Farber MD. Surgical treatment of thyroid-related lower eyelid retraction: a
modified approach. Ophthalmic Plast Reconstr Surg 1992;8: modified

20 Cohen MS, Shorr N. Eyelid reconstruction with hard palate mucosa grafts. Ophthalmic Plast Reconstr Surg 1992;8:18395.

21 Gardner TA, Kennerdell JS, Buerger GF. Treatment of dysthyroid lower lid retraction with autogenous tarsus transplants. Ophthalmic Plast Reconstr Surg 1992;8:26-31.

22 Kushner BJ. A surgical procedure to minimise lower-eyelid retraction with inferior rectus recession.. Arch Ophthalmol 1992;110:1011-4.

23 Pacheco EM, Guyton DL, Repka MX. Changes in eyelid position accompanying vertical rectus muscle surgery and prevention of lower lid retraction with adjustable surgery. $\mathcal{f}$ Pediatr Ophthalmol Strabismus 1992;29:265-72.
24 Shaw GY, Khan J. The management of ectropion using the tarsoconjunctival composite graft. Arch Otolaryngol Head Neck Surg 1996;122:51-5.

25 Whitehouse GM, Francis IC. Eyelid reconstruction using a monopedicle flap and buccal mucosa: report of 15 cases. Aust NZ F Ophthalmol 1988;16:295-301.

26 Hawes MJ, Dortzbach RK. The microscopic anatomy of the lower eyelid retractors. Arch Ophthalmol 1982,10:1313-8.

27 Goldberg RA, Lufkin R, Farahani K, Wu JC, Jesmanowicz A, Hyde JS. Physiology of the lower eyelid retractors: tight linkage of the anterior capsulopalpebral fascia demonstrated using dynamic ultrafine surface coil MRI. Ophthalmic Plast Reconstr Surg 1994;10:87-91.

28 Khaw PT, Sherwood MB,MacKay SLD, Rossi MB, Schultz GS. 5-Minute treatments with fluorouracil, floxuridine and mitomycin have long-term effects on human Tenon's capsule fibroblasts. Arch Ophthalmol 1992;110:1150-4.

29 Khaw PT, Doyle JW, Sherwood MB, et al. Prolonged localised tissue effects from 5-minute exposures to fluorouracil and mitomycin C. Arch Ophthalmol 1993,111:263-7.

30 Sackett DL, Richardson WS, Rosenberg W, Haynes RB. Introduction: on the need for evidence-based medicine. In: Evidence-based medicine: how to practice and teach EBM. 1st ed. New York: Churchill Livingstone, 1997:4-5.

31 Brooks SE, Tibeiro G deB, Archer SM, Elner VM, Del Monte MA. Fat adherence syndrome treated with intraopMonte MA. Fat adherence syndrome treated with intraop-
erative mitomycin-C: a rabbit model. $f$ Pediatr Ophthalmol Strabismus $1996,33: 21-7$.

32 Rubinfield RS, Pfister RP, Stein RM, et al. Serious complications of topical mitomycin C after pterygium surgery. Ophthalmology 1992,99:1647-54

33 Aiache AE, Ramirez OH. The sub-orbicularis oculi fat pads: an anatomic and clinical study. Plast Reconst Surg 1995;95: $37-4$. 